\title{
Placental microRNA Expression Is Not Altered by Maternal Obesity and Fetal Overgrowth
}

\author{
Neda Ghaffari, MD ${ }^{1} \quad$ Samuel Parry, MD ${ }^{1} \quad$ Michal A. Elovitz, MD ${ }^{1} \quad$ Celeste P. Durnwald, MD ${ }^{1}$ \\ ${ }^{1}$ Department of Obstetrics and Gynecology, Center for Research on \\ Reproduction and Women's Health, Maternal and Child Health \\ Research Program, University of Pennsylvania, Perelman School of \\ Address for correspondence Neda Ghaffari, MD, Obstetrix Medical \\ Group of San Jose, 900 E. Hamilton Ave \# 220, Campbell, CA 95008 \\ (e-mail: nedasd@gmail.com).
} Medicine, Philadelphia, Pennsylvania

Am J Perinatol Rep 2016;6:e430-e435.

\begin{abstract}
Keywords

- obesity

- fetal metabolic programming

- microRNA

- macrosomia

Objective The epigenetic mechanisms underlying fetal metabolic programming are poorly understood. We studied whether obesity is associated with alterations in placental miRNA expression.

Study Design A cross-sectional study was performed, including (1) normal-weight women (BMI $\left.20-24.9 \mathrm{~kg} / \mathrm{m}^{2}\right)$ and normal-birth-weight (BW) infants $(2,700-3,500 \mathrm{~g})$ ( $n=20)$, (2) normal-weight and macrosomic infants (BW $\geq 4,000 \mathrm{~g})(n=10)$, (3) obese (BMI $\left.\geq 35 \mathrm{~kg} / \mathrm{m}^{2}\right)$ and normal BW infants $(n=16)$, and (4) obese and macrosomic infants $(n=10)$. All had term deliveries (37-41 weeks) and normal glucose tolerance ( 1 hour GCT $<7.2 \mathrm{mmol} / \mathrm{L}[130 \mathrm{mg} / \mathrm{dL}]$ ). The expression of 5,639 placental miRNAs was assessed using miRNA microarray. Differential miRNA expression was determined using two-way ANOVA and pairwise contrasts, with the Benjamini-Hochberg (BH) correction. MiRNAs with Z-scores $\geq 2$ and false discovery rate (FDR) $<20 \%$ were considered significant.

Results Principal components analysis demonstrated similar global miRNA expression profiles among groups. Of 5,639 miRNAs, only 5 were significantly different between obese and controls, which were not validated by quantitative polymerase reaction. Conclusion There was no difference in placental miRNA expression associated with obesity or overgrowth. Aberrant placental miRNA expression is an unlikely mechanism underlying fetal metabolic programming related to maternal obesity.
\end{abstract}

In the United States, more than one-half of pregnant women are obese or overweight. ${ }^{1}$ These women are at increased risk of adverse perinatal outcomes including fetal overgrowth. ${ }^{2}$ Exposure to maternal obesity or high birth weight predisposes offspring to obesity, hypertension, type 2 diabetes, and cardiovascular disease later in life through fetal metabolic programming. ${ }^{3-7}$ It is accepted that an obesogenic intrauterine environment has long-lasting effects on the fetus, yet the molecular mechanisms contributing to these processes are poorly understood.

Several mechanisms have been explored to elucidate the pathways of fetal metabolic programming, including altered organ development, cellular signaling responses, and epigenetic modifications. ${ }^{8,9}$ Epigenetic modifications underlying fetal programming include transcriptional regulation through DNA methylation, histone modification, and posttranscriptional regulation through microRNAs (miRNAs). ${ }^{10}$

A single miRNA can bind multiple mRNA transcripts, and each mRNA can be bound to multiple miRNAs, allowing miRNAs to modulate entire gene networks. ${ }^{8}$ As such, miRNAs modulate processes such as development, stem cell differentiation, hematopoiesis, muscle development, insulin secretion, cholesterol metabolism, and immune response. ${ }^{9}$ Given their role in developmental processes, miRNAs are natural received

September 15, 2016 accepted after revision November 15, 2016
DOI http://dx.doi.org/ $10.1055 / \mathrm{s}-0036-1597652$. ISSN 2157-6998.
Copyright (c) 2016 by Thieme Medical Publishers, Inc., 333 Seventh Avenue, New York, NY 10001, USA. Tel: +1(212) 584-4662.
License terms

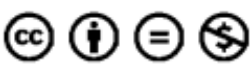


candidate markers of fetal programming in the in utero environment.

While several studies have focused on elucidating fetal metabolic programming in response to overnutrition in animal models, ${ }^{10}$ there is a lack of human studies in this field. The placenta is an accessible biospecimen in humans that is abundant in miRNAs and has been the focus of studies addressing miRNA expression in relation to pregnancy complications, ${ }^{11}$ such as preeclampsia, ${ }^{12,13}$ fetal growth restriction, ${ }^{14,15}$ and preterm labor. ${ }^{16}$ We hypothesized that placental miRNAs involved in regulating nutrient exchange to the fetus would be altered in pregnancies impacted by maternal obesity and fetal macrosomia.

In the present study, our objective was to determine whether an obesogenic in utero environment is associated with specific alterations in placental miRNA profiles as a possible epigenetic mechanism by which maternal obesity might alter fetal programming. We explored the miRNA expression profiles in response to two different surrogates for an in utero environment exposed to overnutrition: maternal obesity and fetal overgrowth.

\section{Methods}

\section{Study Design}

A cross-sectional study was performed in placentas obtained from women with and without maternal obesity and infants born with and without macrosomia. Fifty-six women were prospectively recruited on the labor and delivery unit at the Hospital of the University of Pennsylvania over a 6-month period from January 2013 to July 2013. Inclusion criteria included age 18 to 45 years old, singleton pregnancy, normal glucose tolerance ( 1 hour glucose challenge test [GCT] $<7.2 \mathrm{mmol} / \mathrm{L}$ [130 mg/dL]), and term delivery (37-41 weeks). A GCT threshold of $7.2 \mathrm{mmol} / \mathrm{L}$ was used-the lowest value that has been proposed as a screening cutoff for gestational diabetes mellitus-to best control for any degree of glucose intolerance and study the effect of obesity alone. ${ }^{17}$

Exclusion criteria were based on comorbid conditions that could independently affect fetal growth, including women with chronic hypertension, preeclampsia, chronic steroid use, diabetes, major fetal anomaly, connective tissue disorder requiring medication, and active human immunodeficiency virus (HIV) or hepatitis C.

Women in the following four groups were enrolled: (1) normal maternal weight (body mass index [BMI] $20-24.9 \mathrm{~kg} / \mathrm{m}^{2}$ ) with normal-birth-weight infants $(2,700-3,500 \mathrm{~g})(n=20)$, (2) normal maternal weight with macrosomic infants (birth weight $\geq 4,000 \mathrm{~g})(n=10)$, (3) maternal obesity (BMI $\geq 35 \mathrm{~kg} / \mathrm{m}^{2}$ ) with normal-birth-weight infants $(n=16)$, and (4) maternal obesity with macrosomic infants $(n=10)$.

BMI was calculated at the earliest prenatal visit before 22 weeks to best estimate maternal BMI prior to the influence of pregnancy weight gain. Consistent with the World Health Organization (WHO) classification, normal BMI was defined as a BMI of 20 to $24.9 \mathrm{~kg} / \mathrm{m}^{2}$. For the obese group, a BMI cutoff of $\geq 35 \mathrm{~kg} / \mathrm{m}^{2}$ was selected. Women with obesity classes II and III were selected to ensure that there was a distinct phenotype as compared with the normal-weight group, increasing the likelihood of detecting a difference in miRNA expression from the exposure of maternal obesity.

Macrosomia was defined as a birth weight of $\geq 4,000 \mathrm{~g}$. Normal birth weight between the 10th and 90th percentiles at 37 to 41 weeks of gestation was identified using the Alexander curve: 2,700 to $3,500 \mathrm{~g} .{ }^{18}$ Again, it was intentional that the upper limit of normal birth weight was separated from the cutoff for macrosomia, allowing for distinct phenotypes in the groups.

The sample size was selected based on studies of gene expression microarray showing that 10 to 15 replicates yield results that are stable, with little increase in stability with increasing sample size. ${ }^{19}$ The study was approved by the Institutional Review Board of the University of Pennsylvania. All study participants provided written informed consent.

\section{RNA Isolation}

Placentas were collected immediately after delivery and refrigerated until collection of the samples. A $1 \times 1 \times 1 \mathrm{~cm}$ of placenta was obtained from a central area of the placenta, near the umbilical cord insertion site. There is no standardized method for placental collection ${ }^{20}$; one sample was chosen due to optimize time and budget. An area in the mid-region of the placenta that did not include areas of pathology (i.e., infarcts, calcifications) was selected as a representative sample from each placenta. Samples were washed in cold phosphate-buffered saline (PBS), and stored in $1 \mathrm{~mL}$ Trizole LS. Samples were then stored at $-80^{\circ} \mathrm{C}$ until further use. Total RNA was isolated from the placental samples using Qiagen miRNeasy Serum Kits (Qiagen, Valencia, CA) according to the manufacturer's instructions. Briefly, tissue samples were homogenized using QIAzol Lysis Reagant, and RNA was extracted by phenol/chloroform extraction, followed by silica-membrane-based purification of total RNA. RNA concentration was determined with the NanoDrop spectrophotometer (NanoDrop 2000 Spectrophotometer; Nanodrop, Rockland, DE).

\section{Microarray Array Methods and Analyses}

Affymetrix GeneChip miRNA 3.0 arrays (Affymetrix, Santa Clara, CA) were used to determine miRNA expression, according to the manufacturer's instructions. Briefly, total RNA was labeled using the FlashTag Biotin HSR RNA labeling procedure. A tailing reaction was performed followed by ligation of the biotinylated signal molecule to the target RNA sample. The labeled samples were hybridized to the Affymetrix GeneChip miRNA 3.0 array and scanned according to the manufacturer's instructions. Hybridization images were then scanned and digitized with the Genechip Scanner 3000 (Affymetrix). The normalized signal intensity was $\log 2$ transformed, and data analysis was performed with the Partek 6.6 Genomic Suite software (Copywrite; Partek Inc, St. Louis, MO). The expression of 5,639 human miRNAs was assessed using the Affymetrix GeneChip miRNA 3.0 array. Differential miRNA expression was determined using two-way ANOVA (analysis of variance) and pairwise contrasts, with the Benjamini-Hochberg $(\mathrm{BH})$ correction. MiRNAs associated with 
absolute Z-scores $\geq 2$ or $\mathrm{BH}<0.2$ were considered significant. Global sample variation was assessed by principal components analysis (PCA).

\section{MicroRNA Array Validation by Real-Time Polymerase Chain Reaction}

To confirm miRNA array results when significant differences were observed between two groups, quantitative PCR was performed using stored placental RNA samples from groups 2 (normal-weight women with macrosomic infants) and 4 (obese women with macrosomic infants). Validation of the miRNA array was performed with SYBR Green real-time polymerase chain reaction (qPCR) (Life Technologies, Carlsbad, CA). Briefly, complementary DNA was synthesized with the miScript II Reverse Transcription Kit (Qiagen, Valencia, CA), per the manufacturer's instructions. SYBR Green-based miScript Primer assays were performed using miRNA-specific primers (Qiagen). qPCRs were completed, in triplicate wells on the Applied Biosystems 7900HT Sequence Detection System (Life Technologies) according to the manufacturer's protocol. The DDCT method was used for relative expression quantification with the use of RQ manager software (version 2.4; Life Technologies). ${ }^{21}$ The endogenous reference gene RNU6B (MS00014000) was used for miRNA quantification. Primer sets were purchased from Qiagen: miR-3074-5p and miR-4529-3p.

\section{Statistical Analysis}

For demographic data and clinical characteristics, statistical analyses were performed using STATA version 10.1 (StataCorp LP, College Station, TX). Categorical data were compared using chi-square test. Means and medians of continuous data were compared using ANOVA (for parametric continuous data) and Kruskal-Wallis (for nonparametric continuous data).

\section{Results}

Clinical characteristics of the study groups are shown in - Table 1. Maternal age, race, and fetal sex were statistically different among groups, while the 1 hour GCT values were similar. The median gestational age at delivery was 39.3 to 40.6 weeks. Although there was a statistical difference in the median gestational age at delivery by about 1 week, this difference is unlikely to be clinically significant. Normalweight women gained significantly more weight than their obese counterparts.

PCA demonstrates that the global miRNA expression profiles were overall similar among all samples (-Fig. 1). Of the 5,639 miRNAs investigated, 5 were significantly different between cases and controls, specifically between groups 2 (normal-weight women with macrosomic infants) and 4 (obese women with macrosomic infants) (-Table 2). A three-way ANOVA was performed to determine whether fetal sex was a confounder; however, no difference was seen. The same five miRNAs were significantly different when fetal sex was used as a variable in the three-way ANOVA.

Of the five miRNAs, two were mature miRNAs. These two miRNAs were selected for qPCR validation given that they were the most likely to be biologically active. The relative expression of these two miRNAs was not significantly different by qPCR in placental samples between groups 2 and 4 (-Table 3). Therefore, these likely represent false-positive results from the microarray analysis.

Additional exploratory analyses were performed regrouping samples by potential confounders, including maternal weight gain, race, and maternal age. No significant differences remained in miRNA expression profiles by PCA after these analyses.

\section{Discussion}

This study found that placental miRNA expression was not significantly different in pregnancies exposed to maternal obesity, fetal macrosomia, or a combination of both exposures. Global miRNA expression profiles were similar among all groups, even with additional exploratory analyses regrouping by maternal weight gain, maternal age, fetal sex, and race.

Table 1 Demographics and clinical characteristics

\begin{tabular}{|c|c|c|c|c|c|}
\hline Variable & $\begin{array}{l}1 \text { (Normal BMI/ } \\
\text { Normal BW) } \\
(n=20)\end{array}$ & $\begin{array}{l}2 \text { (Normal BMI/ } \\
\text { Macrosomia) } \\
(n=10)\end{array}$ & $\begin{array}{l}3 \text { (Obese BMI/ } \\
\text { Normal BW) } \\
(n=16)\end{array}$ & $\begin{array}{l}4 \text { (Obese BMI/ } \\
\text { Macrosomia) } \\
(n=10)\end{array}$ & $p$ Value \\
\hline Age & $26.5 \pm 5.8$ & $32.5 \pm 5.2$ & $25.6 \pm 3.9$ & $26.6 \pm 4.2$ & 0.007 \\
\hline Black race & $8(40)$ & $3(30)$ & $14(87.5)$ & $6(60)$ & 0.026 \\
\hline BMI $\left(\mathrm{kg} / \mathrm{m}^{2}\right)$ & $23.1(21.5-24)$ & $22(20.7-23.2)$ & $40.1(37.1-43.7)$ & $39.3(37.6-41.9)$ & $<0.001$ \\
\hline Weight gain (lb) & $34.1 \pm 12.6$ & $32.5 \pm 7.8$ & $19.1 \pm 13.8$ & $27.7 \pm 18.8$ & 0.013 \\
\hline GA del (wk) & $40.3(39.4-40.9)$ & $40.2(40-40.4)$ & $39.3(39.1-39.7)$ & $40.6(40.3-41)$ & .004 \\
\hline Birth weight (g) & $3,204.6 \pm 185.5$ & $4,298.5 \pm 120.9$ & $3,178.5 \pm 159.1$ & $4,191 \pm 218.7$ & $<0.001$ \\
\hline $1 \mathrm{~h} \mathrm{GCT} \mathrm{(mg/dL)}$ & $90.9 \pm 19.4$ & $107.7 \pm 11.4$ & $99.3 \pm 16.1$ & $101.3 \pm 15.5$ & 0.07 \\
\hline Female infant & $12(60)$ & $3(30)$ & $11(68.75)$ & $1(10)$ & 0.012 \\
\hline
\end{tabular}

Abbreviations: BMI, body mass index; BW, birth weight; GCT, glucose challenge test.

p Values determined by chi-square test (categorical data), appropriate comparisons of means and medians using ANOVA (for parametric continuous data) and Kruskal-Wallis (for nonparametric continuous data).

Data are $n(\%)$, median (IQR) or mean \pm standard deviation. 


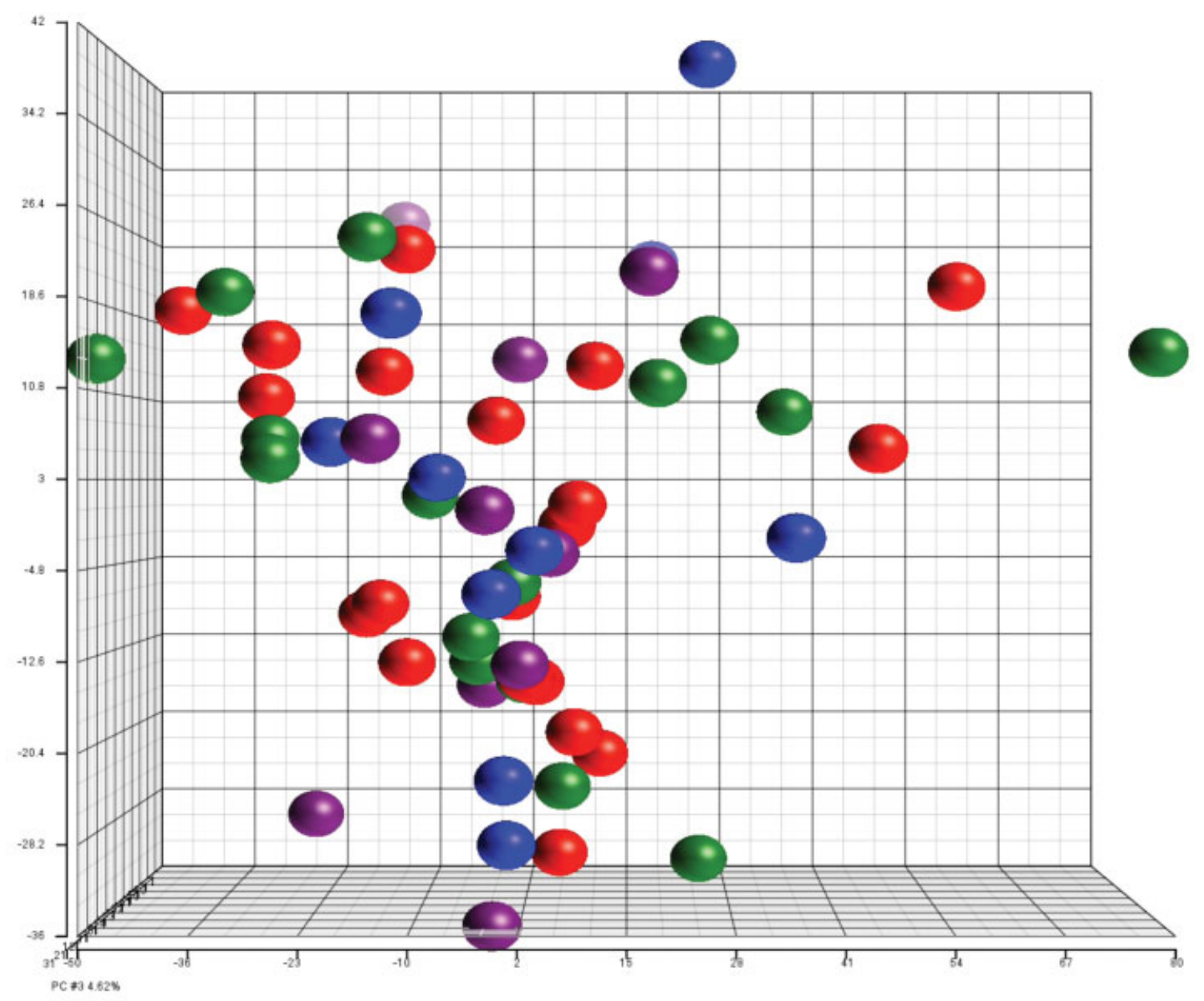

\section{: 1 Nl BMI/Nl bw}

\section{: 2 Nl BMI/macro}

\section{: 3 Obs BMI/NI bw}

\section{: 4 Obs BMI/macro}

Fig. 1 Principal components analysis plot for placental miRNA expression.

Table 2 Significant microRNA array results between group 4 (obese women with macrosomic infants) versus group 2 (normal-weight women with macrosomic infants)

\begin{tabular}{|l|l|l|l|}
\hline Transcript ID & Fold change $^{\mathrm{a}}$ & FDR & Structure \\
\hline mir-3620-st & 2.3 & 0.07 & Stem-loop \\
\hline mir-4529-3p-st & -2.2 & 0.09 & Mature \\
\hline mir-1184-st & -2.4 & 0.05 & Stem-loop \\
\hline mir-103b-st & -2.5 & 0.07 & Stem-loop \\
\hline mir-3074-5p-st & -2.8 & 0.01 & Mature \\
\hline
\end{tabular}

Table 3 Validation of array findings by quantitative polymerase change reaction

\begin{tabular}{|l|l|l|l|}
\hline Transcript ID & $\begin{array}{l}\text { Group 2 } \\
\text { mean RQ }\end{array}$ & $\begin{array}{l}\text { Group 4 } \\
\text { mean RQ }\end{array}$ & p Value \\
\hline mir-4529-3p-st & 2.7 & 3.4 & 0.44 \\
\hline mir-3074-5p-st & 2.0 & 2.0 & 0.85 \\
\hline
\end{tabular}

Abbreviation: FDR, false discovery rate.

${ }^{\mathrm{a}}$ Fold change in miRNA expression between groups 4 and 2 .

Abbreviation: RQ, relative quantification. 
In our analyses, important confounding factors were intentionally excluded, such as glucose intolerance and hypertensive disorders, in an effort to study the effects of maternal obesity in isolation. However, excluding other aspects of the metabolic syndrome may have selected an obese population without sufficient metabolic changes to cause significant epigenetic modifications in the placenta. The effect of other comorbidities on miRNA expression and neonatal outcomes would require further investigation.

Only a few studies have investigated the placental response to an obesogenic environment. Challeir et al discovered increased levels of mRNA expression of macrophage markers and inflammatory cytokines in placental macrophages from obese pregnant women as compared with lean controls. ${ }^{22}$ Differential miRNA expression has also been described in the placenta from pregnancies complicated by fetal growth restriction, suggesting that placental miRNAs are involved in regulating fetal growth. ${ }^{15}$ Animal studies have investigated organ-specific changes in the miRNA profile of offspring after exposure to maternal obesity, including miRNA isolated from baboon hearts, ${ }^{23}$ sheep livers, ${ }^{24}$ and sheep muscle miRNA. ${ }^{25}$

In contrast to these studies, our study did not find miRNA changes in relation to an obesogenic environment in placental samples. Our prior work also demonstrated no miRNA differences in relation to maternal obesity in umbilical cord blood samples. ${ }^{26}$ Our negative findings may be accounted for by the isolation of miRNA from the placenta rather than directly from the fetal organs that may be involved in metabolic programming, such as the liver or pancreas. Additionally, microarray technology may have limited our ability to detect small differences in miRNA expression, as microarray is known to have a lower dynamic range than reverse transcription PCR (RT-PCR). ${ }^{27}$

It is plausible that changes in the miRNA profile occur earlier in pregnancy and are not evident at term. It is known that there is likely a temporal nature to fetal programming. The developing organism passes through multiple critical periods of development at different stages, and the vulnerability of organ systems to certain insults differs according to its stage of development at the time.

Our findings suggest that maternal obesity and fetal overgrowth do not significantly alter the miRNA profile in the fetus, and hence this epigenetic modification is not a key mechanism in adverse outcomes associated with overnutrition. Recent studies suggest an alternative pathway of altered DNA methylation in umbilical cord blood associated with maternal obesity. ${ }^{28,29}$ Alterations in methylation of histones and chromatin remodeling are also being investigated as epigenetic contributors to fetal metabolic programming. ${ }^{30}$

In terms of defining obesity, we used recorded BMI at first prenatal visit to select cases of obesity because prepregnancy weight was self-reported, which has led to misclassification bias in prior studies. ${ }^{31} \mathrm{~A}$ majority of patients in our study presented for prenatal care in the first trimester, so the initial recorded BMI likely accurately reflects their classification prior to weight gain from pregnancy. However, prepregnancy BMI, maternal diet, or weight gain may have a greater effect on fetal programming than obesity alone. The definition of fetal macrosomia is also not well established and there is no known fetal weight cutoff at which long-term offspring health outcomes are elevated. ${ }^{32}$ To assess the impacts of maternal diet or alternative fetal weight cutoffs and miRNA profiling, a more intense study would be required that was beyond the scope of this work.

This study was well designed to discover miRNA differences associated with maternal obesity or fetal macrosomia, and the combination of these factors. The sample size was sufficient for a discovery study, based on studies of gene expression microarray showing that 10 to 15 replicates yield results that are quite stable, and there is less improvement in stability as the number of replicates is further increased. ${ }^{19} \mathrm{An}$ increase in sample size would unlikely change the results.

A limitation to this study design is that we performed a discovery study rather than targeting-specific molecular pathways. A discovery study was selected for this work given the lack of human data on this topic. While we expected changes in the placenta in response to fetal programming of obesity, these changes may be present only in fetal target tissues. Traditionally, the placenta has been used in studies of pregnancy-related diseases and is a feasible human biospecimen.

Our study suggests that fetal metabolic programming in obesogenic pregnancies is unlikely to be caused by alterations in placental miRNA expression at term. Future research should focus on human cohorts with differences in maternal weight gain, maternal diet, maternal visceral adiposity, or alternative definitions of fetal overgrowth. Further investigation is paramount to elucidating the mechanisms underlying the molecular pathways in states of overnutrition, as these processes are known to have important consequences on the health of future generations.

\section{Note}

The Maternal and Child Health Research Program within the Department of Obstetrics and Gynecology at the University of Pennsylvania Health System provided financial support for this research.

\section{Conflict of Interest}

The authors report no potential conflicts of interest.

\section{References}

1 American College of Obstetricians and Gynecologists. ACOG Committee opinion no. 549: obesity in pregnancy. Obstet Gynecol 2013;121(1):213-217

2 Catalano PM, Hauguel-De Mouzon S. Is it time to revisit the Pedersen hypothesis in the face of the obesity epidemic? Am J Obstet Gynecol 2011;204(6):479-487

3 Barker DJ, Gluckman PD, Godfrey KM, Harding JE, Owens JA, Robinson JS. Fetal nutrition and cardiovascular disease in adult life. Lancet 1993;341(8850):938-941

4 Mamun AA, Hayatbakhsh MR, O'Callaghan M, Williams G, Najman J. Early overweight and pubertal maturation-pathways of association with young adults' overweight: a longitudinal study. Int J Obes 2009;33(1):14-20 
5 Wen X, Triche EW, Hogan JW, Shenassa ED, Buka SL. Prenatal factors for childhood blood pressure mediated by intrauterine and/or childhood growth? Pediatrics 2011;127(3):e713-e721

6 Lau C, Rogers JM, Desai M, Ross MG. Fetal programming of adult disease: implications for prenatal care. Obstet Gynecol 2011; 117(4):978-985

7 Boney CM, Verma A, Tucker R, Vohr BR. Metabolic syndrome in childhood: association with birth weight, maternal obesity, and gestational diabetes mellitus. Pediatrics 2005;115(3):e290-e296

8 Kappil M, Chen J. Environmental exposures in utero and microRNA. Curr Opin Pediatr 2014;26(2):243-251

9 Williams AE. Functional aspects of animal microRNAs. Cell Mol Life Sci 2008;65(4):545-562

10 Zhang J, Zhang F, Didelot X, et al. Maternal high fat diet during pregnancy and lactation alters hepatic expression of insulin like growth factor- 2 and key microRNAs in the adult offspring. BMC Genomics 2009;10:478. doi: 10.1186/1471-2164-10-478

11 Zhao Z, Moley KH, Gronowski AM. Diagnostic potential for miRNAs as biomarkers for pregnancy-specific diseases. Clin Biochem 2013;46(10-11):953-960

12 Luo R, Shao X, Xu P, et al. MicroRNA-210 contributes to preeclampsia by downregulating potassium channel modulatory factor 1 . Hypertension 2014;64(4):839-845

13 Kleinrouweler CE, van Uitert M, Moerland PD, Ris-Stalpers C, van der Post JA, Afink GB. Differentially expressed genes in the preeclamptic placenta: a systematic review and meta-analysis. PLoS One 2013;8(7):e68991

14 Pineles BL, Romero R, Montenegro D, et al. Distinct subsets of microRNAs are expressed differentially in the human placentas of patients with preeclampsia. Am J Obstet Gynecol 2007;196(3): 261.e1-261.e6

15 Maccani MA, Padbury JF, Marsit CJ. miR-16 and miR-21 expression in the placenta is associated with fetal growth. PLoS One 2011; 6(6): 221210

16 Mayor-Lynn K, Toloubeydokhti T, Cruz AC, Chegini N. Expression profile of microRNAs and mRNAs in human placentas from pregnancies complicated by preeclampsia and preterm labor. Reprod Sci 2011;18(1):46-56

17 Donovan L, Hartling L, Muise M, Guthrie A, Vandermeer B, Dryden DM. Screening tests for gestational diabetes: a systematic review for the U.S. Preventive Services Task Force. Ann Intern Med 2013; $159(2): 115-122$

18 Alexander GR, Himes JH, Kaufman RB, Mor J, Kogan M. A United States national reference for fetal growth. Obstet Gynecol 1996;87(2):163-168
19 Pavlidis P, Li Q Noble WS. The effect of replication on gene expression microarray experiments. Bioinformatics 2003; 19(13):1620-1627

20 Burton GJ, Sebire NJ, Myatt L, et al. Optimising sample collection for placental research. Placenta 2014;35(1):9-22

21 Livak KJ, Schmittgen TD. Analysis of relative gene expression data using real-time quantitative PCR and the 2(-Delta Delta $\mathrm{C}(\mathrm{T})$ ) Method. Methods 2001;25(4):402-408

22 Challier JC, Basu S, Bintein T, et al. Obesity in pregnancy stimulates macrophage accumulation and inflammation in the placenta. Placenta 2008;29(3):274-281

23 Maloyan A, Muralimanoharan S, Huffman S, et al. Identification and comparative analyses of myocardial miRNAs involved in the fetal response to maternal obesity. Physiol Genomics 2013;45(19): 889-900

24 Nicholas LM, Rattanatray L, MacLaughlin SM, et al. Differential effects of maternal obesity and weight loss in the periconceptional period on the epigenetic regulation of hepatic insulin-signaling pathways in the offspring. FASEB J 2013;27(9):3786-3796

25 Yan X, Huang Y, Zhao JX, et al. Maternal obesity downregulates microRNA let-7g expression, a possible mechanism for enhanced adipogenesis during ovine fetal skeletal muscle development. Int J Obes 2013;37(4):568-575

26 Ghaffari N, Parry S, Elovitz MA, Durnwald CP. The effect of an obesogenic maternal environment on expression of fetal umbilical cord blood miRNA. Reprod Sci 2014. doi: 10.1177/ 1933719114565032

27 Draghici S, Khatri P, Eklund AC, Szallasi Z. Reliability and reproducibility issues in DNA microarray measurements. Trends Genet 2006;22(2):101-109

28 Gemma C, Sookoian S, Alvariñas J, et al. Maternal pregestational BMI is associated with methylation of the PPARGC1A promoter in newborns. Obesity (Silver Spring) 2009;17(5):1032-1039

29 Perkins E, Murphy SK, Murtha AP, et al. Insulin-like growth factor 2/H19 methylation at birth and risk of overweight and obesity in children. J Pediatr 2012;161(1):31-39

30 Sookoian S, Gianotti TF, Burgueño AL, Pirola CJ. Fetal metabolic programming and epigenetic modifications: a systems biology approach. Pediatr Res 2013;73(4 Pt 2):531-542

31 Bodnar LM, Siega-Riz AM, Simhan HN, Diesel JC, Abrams B. The impact of exposure misclassification on associations between prepregnancy BMI and adverse pregnancy outcomes. Obesity (Silver Spring) 2010;18(11):2184-2190

32 Rossi AC, Mullin P, Prefumo F. Prevention, management, and outcomes of macrosomia: a systematic review of literature and meta-analysis. Obstet Gynecol Surv 2013;68(10):702-709 